\title{
Pour une poésie de circonstance
}

\section{La venue du poème}

Dans l'œuvre d'Henry Bauchau, il n'y a peut-être pas, à proprement parler, de temps pour la poésie, tant celle-ci, loin de n'occuper qu'une phase initiale - celle des vers de jeunesse, dont témoigne le "Cantique de l'attente " écrit " dans un état d'inspiration soudaine " en 1932, ou encore celle du premier livre publié, Géologie, en 1958 -, apparaît comme une résurgence aussi intermittente que permanente, l'écriture poétique coexistant sans cesse avec des formes romanesque, théâtrale ou diariste qu'elle provoque, accompagne ou prolonge.

Pour autant, la création poétique semble obéir à une temporalité particulière. Dans sa "Lecture " d'Heureux les déliants, Geneviève Henrot a fixé les étapes de cette genèse du poème propre à Henry Bauchau : "la fonction génétique du signifiant (sonore, vocal, rythmique), la fulguration de l'ébauche initiale autour de "constellations impérieuses", la nécessité, ensuite, d'une lente maturation [...] et, enfin, l'exigence d'exhaustivité dans la confession "². L'émergence de la poésie est en particulier vécue sur un mode contradictoire, entre l'éclatement et la continuité, entre le déferlement du verbe et sa maturation silencieuse, entre l'irruption d'une parole impérative et le patient travail sur la matière du langage. Les journaux, en particulier, témoignent fréquemment de ce temps alterné de venue, de repos et de labeur, saisissant volontiers le premier état de la parole poétique avant d'évoquer parfois les étapes des rédactions ultérieures ou les modifications entraînées par les conseils de proches lecteurs ${ }^{\mathbf{3}}$. Et Henry Bauchau lui-même, dans "Dépendance amoureuse du poème ", met en évidence la double temporalité créatrice qui préside à son écriture poétique : d'abord le temps fort de l'inspiration ("Survient un son, un rythme, une image, une intuition et j'ai soudain le désir, l'espérance d'écrire un poème »- PC, p. 8), puis le temps faible du labeur et de l'attente ("C'est le moment de la patience, de la ténacité, d'un travail qui semble devenu vain ", PC, p. 8), cet en-avant comme cet enlisement s'avérant tous deux nécessaires à la naissance du poème, fruit dialectique de l'association du jour et de la nuit, du conscient et de l'inconscient.

Cette marche heurtée du poème, qui procède par saccades et par relances et où la stagnation même peut s'avérer un progrès, n'est pas sans conséquences sur

1 JA, 21 novembre 1989, p. 16. Les premières ébauches poétiques datées des années 1930 ont par ailleurs été dévoilées et étudiées dans Geneviève Duchenne, Vincent Dujardin \& Myriam WattheeDelmotte, Henry Bauchau dans la tourmente du xx siècle. Configurations historiques et imaginaires, Bruxelles, Le Cri, "Biographie ", 2008, p. 131-144.

2 Geneviève Henrot, "Lecture ", dans HD, p. 340.

3 Sur cette question, voir Catherine Mayaux, "La dictature du poème, ou le poème, cet inconnu. L'expérience poétique chez Henry Bauchau d'après ses journaux ", dans $N u(e)$, n 35, mars 2007, p. $199-210$. 
l'existence de l'auteur. De fait, Henry Bauchau souligne combien la gestation des poèmes vient rompre le cours du quotidien, détruisant la progression cumulative et linéaire des jours du calendrier au profit d'une durée affolée, débridée, exaltée - temps d'exception qui, ne pouvant devenir une règle, ruine toute régularité. "Dépendance amoureuse du poème " le dit d'ailleurs avec netteté : "La poésie dévaste la vie courante, elle la dénude, elle déborde le poète. " (PC, p. 8). Point de vue auquel l'auteur de Tentatives de louange se montre encore fidèle dans un texte daté d'octobre 2010, plaçant l'exercice de la poésie sous le signe de la dislocation, là où le roman impose au contraire sa charge dans la continuité : "Tu n'es que le locataire de la maison de l'écriture, quand tu es chevauché par le roman ou disloqué par le poème. " Cette violence disruptive de la poésie, Henry Bauchau n'est du reste pas le seul à l'éprouver, à l'exemple de René Char qui évoquait dans La Parole en archipel "l'énergie disloquante de la poésie " 5 .

Face à une telle force, la passivité même du poète est un acte - l'état de réceptivité et de disponibilité aux sollicitations de l'inconscient fournissant le terreau du poème. On comprend, dans ces conditions, que le terme de création ne rende qu'imparfaitement compte du processus de l'écriture poétique. Une page du Journal d'Antigone l'indique, notant que la "création, au sens de la Genèse, se fait à un second stade " de la gestation de l'œuvre, le premier temps consistant à "laisser monter en soi ce qui vient d'une source inconnue et qui a sans doute son origine dans le toute petite enfance. " (JA, 25 novembre 1989, p. 17). Plus loin, la métaphore hydrologique reparaît pour caractériser plus nettement encore la dynamique syncopée de la poésie : «Pour les moments où le flot passe et déborde dans l'inspiration, il faut les longues heures de préparation, de travail obscur, affronter les turbulences des courants et les risques d'inondation. " (JA, 20 juin 1993, p. 254). Débordement ravageur et remous souterrain, la poésie telle que l'évoque Henry Bauchau conjoint ainsi l'enfouissement et le déferlement, s'infiltrant dans le quotidien à coups de jaillissements nocturnes tout autant que par un lent travail de sape, et réclamant moins, au fond, un auteur actif qu'un récepteur attentif. C'est pourquoi, dans cette économie paradoxale de la création poétique, ne pas écrire possède aussi une vertu positive.

Rien de plus significatif, à cet égard, que la confidence qui ouvre le poème "La cressonnière "(dans Les Deux Antigone, PC, p. 271) : "Le poème veut un temps d'attente et de refus, un temps d'accueil ". Le moment de la réticence et celui de la confiance se trouvent ainsi juxtaposés, sans que l'on sache exactement s'ils sont conjoints ou successifs. L'indécision logico-temporelle, pourtant, doit être maintenue - précisément parce que le poème se situe moins dans la mesure du temps que dans sa dé-mesure, son excès ou son défaut. C'est du moins dans cette perspective que l'on peut lire l'adjonction qui conclut ce verset initial en disant du poème qu'« il

4 Henry Bauchau, "Le salut au soleil ", dans TL, p. 17.

5 René Char, "Pour un Prométhée saxifrage ", dans La Parole en archipel, Euvres complètes, Paris, Gallimard, "Bibliothèque de la Pléiade ", 1995, p. 399. 
est aussi la circonstance ": définition qui érige le texte poétique en réceptacle d'une histoire (personnelle ou collective) autant qu'en événement fondateur d'une époque, au sens où le grec ancien fait de l'épochè une suspension marquant le point de départ d'une nouvelle ère. À l'échelle de "La cressonnière ", ce basculement dans le temps du poème correspond à l'émergence du langage de l'inconscient, lorsque le sujet lyrique cherche à expliquer l'apparition du syntagme " un os tout nu ", que son oreille "avait perçu un ouf tout nu ": de l'os à l'œuf, du symbole de la mort à celui de la naissance, le texte procède alors à une remontée vers l'origine de la parole, interrogeant tour à tour la figure du roi Saül " couché nu aux pieds du vieillard Samuel, prophétisant tout un jour, toute une nuit, sans pénétrer dans l'Écriture ", puis le motif de cette cressonnière effacée "par les pelles mécaniques ", source " ovale » où l'enfant traçait dans le sable des signes détruits eux aussi.

\section{Le temps de la circonstance}

En définissant le poème non comme un texte de circonstance mais comme la circonstance même, "La cressonnière " met le doigt sur un élément déterminant de la temporalité créatrice. Car en devenant circonstance, le poème n'est plus simplement affecté par son contexte ; il parvient à imposer son temps, un temps astreignant, capricieux, qui oblige le poète à vivre en fonction des exigences du texte et à se tenir sans cesse autour de lui (on retrouve ici le sens étymologique du latin circumstare), comme un satellite tournant autour d'un astre à la fois proche et étranger. Soleil attirant autant qu'inaccessible, telle est bien, du reste, l'image du poème dans ce passage de "L'innocence de l'oreille " (EE, p. 148) : "Je m'approche de sa circonstance, de ce qu'il est, mais je ne l'atteins pas, il m'éclaire de ses rayons, je ne suis pourtant que son traducteur, il ne vient pas de mon être et ne fera jamais partie de mon avoir car il n'est pas privatisable. "

Le lien du poème à la circonstance est l'un des traits fondamentaux de la poétique d'Henry Bauchau, que Geneviève Henrot range même sous le " commandement de la circonstance ", rappelant au passage que l'écrivain a songé à ce mot-clé pour rassembler ses poèmes, avant de renoncer de peur " de ne pas être compris " ${ }^{6}$. Le terme de circonstance n'en a pas moins trouvé sa place en tête de plusieurs textes à la portée réflexive. Henry Bauchau, en 1988, réunit par exemple sous le titre L'Écriture et la circonstance quatre conférences données à la Chaire de Poétique de l'Université de Louvain-la-Neuve - le premier de ces quatre essais étant lui-même nommé "La circonstance éclatante ".

"La circonstance "fournit également le titre de deux poèmes. Le premier est celui qui clôt L'Escalier bleu (PC, p. 101). Ce texte personnifie différents éléments d'un paysage hivernal sous les traits d'une figure tutélaire de l'enfance : les vallées prennent la taille de "l'épousée " sous le manteau de la " Neige mince de novembre ", les arbres deviennent des "athlètes sans ornements ", les mélèzes

6 Geneviève Henrot, Henry Bauchau poète. Le vertige du seuil, Genève, Droz, 2003, p. 20. 
donnent leur forme à "La robe miraculée / De l'ange ", et les greniers laissent entrer un hiver dont le "tablier / Porte les années d'enfance ". En contrepoint de cette musique heureuse, évoquant un passé harmonieusement retenu dans les mailles du décor alpin, le poème s'achève par une invocation au " Léger soleil déclinant " de l'hiver, rayon de vie au seuil de la vieillesse : "Fais que nous soyons ainsi // Simples fermes recueillis / Lorsque nous serons aussi / Déchus de la circonstance ". Paradoxe de la circonstance : cette souveraineté heureuse a beau être éphémère, c'est précisément parce qu'elle s'éprouve dans la perte qu'elle commande la fidélité à son souvenir. Il s'agit au fond d'un moment privilégié, d'un accord subtil entre l'homme et les éléments, d'une grâce peut-être, qui ne s'obtient que dans la ferveur d'un instant ou d'une saison, et dont le sujet s'engage à ne pas démériter quand bien même elle lui serait retirée.

C'est aussi sous la bannière de "La circonstance " que se range le dernier texte d'Heureux les déliants ${ }^{7}$, qui récapitule en trois vers un autre paradoxe, celui d'un poème lesté du poids de son contexte, mais ouvert à tous vents et à l'appropriation des lecteurs : "Prisonnier d'un homme et d'un temps / Enfermé dans ma langue et le réseau de mes images / Je suis à tous, dit le poème, comme le ciel. "Quant à la circonstance, elle semble ici constituer à la fois le moment et le mouvement permettant la cristallisation de cette alliance des contraires en quoi consiste le poème.

Cette allégeance à la circonstance invite à situer la poétique d'Henry Bauchau en regard d'une revendication littéraire présente au moins depuis les propos fameux de Goethe, tels qu'ils ont été recueillis par Eckermann : "Mes poèmes sont tous des poèmes de circonstance, ils s'inspirent de la réalité, c'est sur elle qu'ils se fondent et reposent. Je n'ai que faire de poèmes qui ne reposent sur rien. „8 C'est précisément ce passage qui revient à la mémoire d'Henry Bauchau dans une page du Journal d'Antigone en date du 25 décembre 1992 (JA, p. 198) :

Conversation agréable avec un peintre, qui me semble plein de talent et d'intelligence. Il me dit : "J'ai besoin des objets, des lieux, des êtres pour peindre. Je ne peux pas partir de rien ou de mes visions intérieures. "Cela me rappelle la réflexion de Goethe : "Je ne me soucie pas d'écrire des poèmes qui ne reposent sur aucunes circonstances. »

À la lumière conjuguée de la conversation avec le peintre et du dialogue avec Goethe, la circonstance selon Henry Bauchau se comprend comme la garantie d'un ancrage existentiel du poème, comme si l'écriture avait besoin de la butée du réel pour mieux s'assurer de toucher à une vérité - peut-être cette "vérité de parole "dont Yves Bonnefoy a fait le titre de l'un de ses recueils d'essais. De ce point de vue, c'est d'ailleurs dans le sillage de Bonnefoy et d'autres poètes révélés

7 Henry Bauchau, "La circonstance ", dans Heureux les déliants, PC, p. 304. Sans titre et avec quelques différences de ponctuation, ce texte apparaît le 12 avril 1993 dans JA, p. 229.

8 Conversations de Goethe avec Eckermann (18 septembre 1823), traduit de l'allemand par Jean Chuzeville, édition revue par Claude Roëls, Paris, Gallimard, "Du monde entier », 1988, p. 64. 
après la guerre (Jaccottet, Grosjean, Guillevic ou Ponge) que l'on pourrait situer la démarche poétique d'Henry Bauchau, comme le fait Aude Préta-de Beaufort en rappelant que tous ces poètes, au-delà de leur diversité, " enracinent leur quête du sens dans l'ici-et-maintenant, irréductible au concept, de l'expérience sensible "?. Ce rapprochement ne doit pourtant pas occulter la singularité du réel mis en œuvre par Henry Bauchau. Si les poètes mentionnés se sont en effet tournés vers "la réalité rugueuse à étreindre ", selon le mot de Rimbaud ${ }^{\mathbf{1 0}}$, c'est souvent en réaction contre un surréalisme accusé de privilégier l'évanescence des images et de l'onirisme, la voyance ainsi pratiquée perdant de vue une réalité historique que la guerre venait de ruiner et qu'il s'agissait de reconstruire. Henry Bauchau, de son côté, ne renonce pas à la plongée dans les profondeurs de la pensée, non pour en retirer les trésors de quelque automatisme verbal (encore que tel passage du Journal d'Antigone souligne la proximité entre écriture poétique et automatique ${ }^{\mathbf{1 1}}$ ), mais tout simplement pour approcher la vérité d'une origine, poésie et psychanalyse jouant ici une provocation mutuelle et procédant d'une attention analogue aux différentes formes de langage de l'inconscient : rêves, lapsus, phrases de réveil ou de demi-sommeil. C'est pourquoi la circonstance du poème, loin de se restreindre à un référent extralinguistique, peut émerger d'un signifiant apparemment arbitraire (comme cet " os " ou cet " ouf tout nu " que "La cressonnière " tente de déchiffrer en exploitant ses virtualités mythiques et ses souvenirs personnels), ou d'un rythme obsédant (comme " ce vers bref de trois pieds " qui s'est imposé pour l'écriture de "L'inattendu d'avril " et que le poète, comme il le confie dans le Journal d'Antigone, n'a pu " remettre en question " malgré les difficultés occasionnées par sa brièveté ${ }^{\mathbf{1 2}}$ ) qui tiendrait à la fois du "vers donné " tel que l'évoquait Paul Valéry et de ces échos verbaux de l'inconscient dont André Breton attendait qu'ils donnent "le la » à sa poésie ${ }^{\mathbf{1 3}}$.

Ainsi éprouvée comme une voie - et une voix - d'accès au temps mythique et fantasmatique de l'inconscient, la circonstance d'Henry Bauchau se démarque également de la conception d'Aragon, qui se revendique explicitement d'une poésie de circonstance dans "Les poissons noirs ou de la réalité en poésie ", préface de 1946 au Musée Grévin, long poème dénonciateur du régime de Vichy paru en 1943 sous le pseudonyme de François la Colère. Avec cette préface polémique, Aragon reprend à son compte l'expression " poème de circonstance " (que Breton, en 1932, avait assignée en mauvaise part à " Front rouge ", son éloge du régime stalinien), et

9 Aude Préta-de Beaufort, "Henry Bauchau, poète herméneute : exégèse de soi, exégèse du monde ", dans Catherine Mayaux et Myriam Watthee-Delmotte (dir.), Henry Bauchau, écrire pour habiter le monde, Saint-Denis, Presses universitaires de Vincennes, "L'imaginaire du texte ", 2009, p. 39.

10 Arthur Rimbaud, "Adieu ", dans Une Saison en enfer, Euvres complètes, éd. André Guyaux, Paris, Gallimard, "Bibliothèque de la Pléiade », 2009, p. 279.

11 Voir Henry Bauchau, JA, 28 mai 1995, p. 404-405.

12 Voir Ibid., 20 avril 1995, p. 401.

13 Breton a en effet donné ce titre à un dernier recueil qui réunit une poignée de fragments automatiques. Voir André Breton, Le La, dans Euvres complètes, tome IV, Paris, Gallimard, "Bibliothèque de la Pléiade ", 2008, p. 339-344. 
livre plus largement un plaidoyer pour une littérature politique, historique et nationale, telle qu'en réclament les temps de lutte. Aragon s'inscrit en particulier dans le sillage de l'épopée, cette poésie de combat : "Que ce sont les circonstances qui font l'épique de la poésie implique évidemment que l'épopée est toujours poésie de circonstance. "14 Plus loin, il s'appuie sur le mot de Goethe pour affirmer que "les circonstances dominent la poésie vivante "15. La circonstance devient ainsi la justification d'une poésie militante, qui se veut non seulement le témoin mais aussi l'acteur de son temps.

Cette dimension politique du poème, telle qu'elle est revendiquée par Aragon, ne se retrouve pas chez Henry Bauchau, dont la circonstance se tourne plutôt du côté de l'intériorité que de l'actualité. Au fond, c'est surtout la solidarité entre l'histoire personnelle et l'Histoire humaine (ce que Kafka nomme, dans une formule qui a frappé Bauchau, "l'histoire mondiale de ton âme »16), qui conduit certaines pages à être, comme l'écrivait Char de ses Feuillets d'Hypnos, "affectées par l'événement »"17. Si la circonstance historique s'inscrit à plusieurs reprises dans l'œuvre poétique, c'est donc toujours à travers le prisme d'une méditation personnelle qui replace l'événement ponctuel dans une durée au long cours. "Les chars de Budapest " opposent par exemple à la brutalité de la répression soviétique, qui écrase l'insurrection hongroise en novembre 1956, "la connaissance de la douleur " et le "soleil " futur de "la jeunesse du monde " (PC, p. 47). En 1978, le poème introspectif qu'est La Sourde oreille ou Le rêve de Freud se révèle hanté par la montée des totalitarismes au cours des années 1930, ce temps de la jeunesse qui est aussi "le temps d'Hitler et de Staline [...], le temps des ateliers fermés et du chômage ", celui de la " messe apocalyptique " (PC, p. 234) du nazisme et bientôt de "l'Europe barbelée " (PC, p. 240). Puis c'est avec les Poèmes pendant la Guerre du Golfe (parus en 1995 dans Heureux les déliants) que l'écho de l'actualité résonne le plus explicitement dans l'écriture poétique, en particulier avec "Le matin de la Guerre du Golfe ", qui réplique au déferlement de la violence technologique par la simple chaleur d'une main humaine : "Dans la honte, dans la confusion de ce jour / il y a une menue présence / qui ne guide pas, qui n'éclaire pas / qui se contente, légère, d'être là " (PC, p. 287). Si aucune date n'est ici mentionnée, la chronologie événementielle intervient dans la "Petite suite au 11 septembre 2001 " (dans Nous ne sommes pas séparés PC, p. 339-344), qui convoque l'œuvre de Nancy Huston et la parole de l'Évangile dans une méditation sur la destinée de la "Surgissante, surpuissante Amérique " au cours du " long gémissement séculaire " de l'Histoire.

Poésie de circonstance, donc, que celle d'Henry Bauchau - mais pour laquelle la circonstance est finalement moins un événement extérieur que l'événement

14 Louis Aragon, Euvres poétiques complètes, tome I, Paris, Gallimard, "Bibliothèque de la Pléiade ", 2007, p. 917.

15 Ibid., p. 927.

16 Cité par Henry Bauchau dans JA, 23 août 1991, p. 110.

17 René Char, Feuillets d'Hypnos, dans Euvres complètes, op. cit., p. 173. 
même de l'énonciation poétique, qui fait affleurer dans le rythme d'une parole un ensemble de forces contenues à l'état latent dans l'histoire de l'inconscient. Rien d'étonnant, dans ces conditions, à ce que le temps de la poésie bauchalienne - son temps propre, celui qu'elle met textuellement en œuvre, ce temps irréductible au mouvement orienté d'une narration romanesque, de la durée vécue sur une scène de théâtre ou de la chronologie linéaire d'un journal - se serve de l'instant vécu, avec sa charge référentielle, comme de tremplin à une quête des origines qui excède bien souvent tout repère temporel.

\section{De la circonstance à la synthèse des temps}

Si le poème " est aussi la circonstance ", comme le dit "La cressonnière ", alors peut-être la circonstance est-elle ce moment de bascule qui voit naître la parole poétique. La poésie, en ce sens, viendrait mettre en évidence le processus même de l'énonciation qui, comme l'a montré Benveniste, construit le temps à l'aide de ses propres repères, le présent du discours jouant le rôle de point d'origine d'une chronologie à partir de laquelle s'organisent les autres tiroirs verbaux :

On pourrait croire que la temporalité est un cadre inné de la pensée. Elle est produite en réalité dans et par l'énonciation. De l'énonciation procède l'instauration de la catégorie du présent, et de la catégorie du présent naît la catégorie du temps. Le présent est proprement la source du temps. ${ }^{18}$

De manière analogue, le poème bauchalien donne à lire la naissance du temps de la parole poétique, un temps énonciatif qui, parti du point d'ancrage fourni par la circonstance, se dilate en parcourant le présent, le passé et le futur, jusqu'à les englober dans une véritable osmose.

C'est pourquoi la poésie de circonstance telle que l'élabore Henry Bauchau passe souvent par une écriture où l'ancrage spatio-temporel est fortement marqué, en particulier par l'usage de ce que Benveniste appelle « les indicateurs de la deixis, démonstratifs, adverbes, adjectifs, qui organisent les relations spatiales et temporelles autour du "sujet" pris comme repère "19. Ce repérage autour du sujet, qu'il apparaisse sous la forme du Je ou se dédouble en un Tu, organise même parfois tout un cycle de poèmes. La Chine intérieure contient ainsi une série de textes qui, du "Voyage " au "Retour ", quittent " la durée de la neige " (PC, p. 184) et le cadre du paysage alpin de Gstaad pour un passage à Paris. Cette parenthèse vécue dans le temps actif et les obligations de la vie parisienne est rythmée par des poèmes dont l'incipit désigne un sujet toujours en mouvement, balloté d'un point à un autre, cherchant son lieu sans le trouver : "Tu pars, tu vas quitter la durée de la neige " ("Le voyage ", PC, p. 184) ; "Tu apportes un chèque à la banque "

18 Émile Benveniste, "L'appareil formel de l'énonciation ", dans Problèmes de linguistique générale 2, Paris, Gallimard, « Tel », 1980, p. 83.

19 Id., "De la subjectivité dans le langage ", dans Problèmes de linguistique générale 1, Paris, Gallimard, « Tel », 1976, p. 262. 
("La Sibylle ", PC, p. 186) ; "Tu es dans le train qui vient de Milan " ( L'île SaintLouis ", PC, p. 187) ; "Tu vas à Vincennes ce matin " (L'espérance ", PC, p. 189) ; "Je suis dans la maison de la télévision " (Le serpent d'images ", PC, p. 191) ; "Tu es auprès de la Sibylle " ("La science ", PC, p. 192) ; "Tu es dans le bureau de Dominique, près de son divan d'analyste " "L'heure du thé ", PC, p. 194) ; "Je reviens à la gare de Lyon" ("La nuit close ", PC, p. 196) ; "Tu es revenu et la neige tombait, tu es entré dans le nuage " ("Le retour ", PC, p. 197).

La poésie d'Henry Bauchau tend plus généralement à enraciner la parole dans un hic et nunc mis d'emblée en scène par le poème. Tel est le cas de "La grappe " (PC, p. 108), dont le vers initial concentre autour de l'adverbe ici toute la substance du raisin : "Ici le sang l'eau de la mer avec le vin ". Quant au "Matin de la guerre du Golfe " (PC, p. 287), il s'ouvre sur un maintenant qui dresse un constat d'impuissance face à fatalité des armes (" Maintenant qu'il n'y a plus de voie, plus de choix ") et désigne sans le dater " ce jour " qui voit les troupes occidentales attaquer l'Irak. Cette convocation du contexte immédiat est également assurée par des déterminants démonstratifs, dont la valeur déictique est activée par leur position initiale. Le lecteur des deux " Paraboles " de L'Accueil est ainsi invité à entrer de plain-pied dans la double vision du locuteur : "Ce matin à ma fenêtre / Quatre roses endormies " (PC, p. 383) ; "Ce matin à ma fenêtre / Quatre roses qui s'éveillent " (PC, p. 384). Dans "L'ardoise ", cette proximité du décor végétal est inscrite dans la perspective d'un paysage intensément vécu et d'un passé encore lié au présent : "J'ai aimé ce jardin et je l'ai planté d’arbres / Et ce pays de vent je l'ai semé de fleurs robustes " (PC, p. 159).

Poussant plus loin la localisation dans le passé, le présentatif c'était parvient néanmoins à actualiser la force du souvenir à travers la conscience du sujet lyrique : La Sourde Oreille s'ouvre sur un "C'était l'automne " (PC, p. 221) qui renvoie en réalité à la saison de la jeunesse, de même que le début de "Ma jolie " découvre un renouveau de vie au coeur de l'hiver ("C'était la rose de janvier ", PC, p. 391). Avec des effets comparables, le présentatif il y a introduit lui aussi une circonstance qui se donne telle une naissance heureuse, comme au début du " Plaisir " "Il y a / le matin le soleil ", PC, p. 79), ou au contraire comme une évidence accablante, comme dans ce premier vers de "Les yeux dans le noir " qui caractérise ainsi l'existence : "Il y avait la nuit des batailles de dogues " (PC, p. 368). Le il y avait peut aussi s'apparenter au il était une fois des contes pour reconstruire, le temps du poème, le souvenir d'un âge d'or disparu. Tel est le cas des deux évocations du jardin paternel au bord de la Meuse, éden de l'enfance où un cerisier se substitue à l'arbre de la connaissance : "Dans le jardin du père / sur le bord de la Meuse [...] / il y avait / un cerisier / qui était tout / couvert d'amour ", rappelle ainsi "Le cerisier " des Deux Antigone (PC, p. 280), suivi par " Meuse pour Valentine " dans Exercice du matin ("En bord de Meuse il y avait / tout couvert de mémoire / un cerisier qui nous aimait / dans le jardin du père ", PC, p. 309). 
Mais la circonstance poétique d'Henry Bauchau ne se réduit pas à la référence. Elle est même d'autant moins un référent temporel strictement situé qu'elle sert en réalité de porte d'entrée dans une temporalité contradictoire et déréglée, dont le principe paradoxal est mis en place dès "Géologie " : "Tout rajeunit en s'écoulant ", affirme la fin du poème, en appelant à nager dans le " courant " de ce "torrent " temporel venu rompre le cours du "jour très vieux " et de ses "verbes lents " $\mathrm{PC}$, p. 19). Dès lors, l'entrée dans le devenir permet d'envisager la quête de l'origine, tant il est vrai, comme le note Geneviève Henrot, que la poésie d'Henry Bauchau s'efforce "de remonter à la source de la temporalité originaire. »"

De cet originel, l'enfance se révèle l'une des figures de proue. Le septième texte de La sourde Oreille s'ouvre sur une invitation qui dit à la fois l'attrait et le danger de cette voie étroite que représente la quête de l'origine : "Par le tranchant, si tu peux remonter le fil bien aiguisé du temps, jusqu'à l'enfant que tu ne connais plus » (PC, p. 230). Dans cette perspective, on ne s'étonnera pas que des recueils comme Géologie ou L'Escalier bleu, nourris par l'expérience de l'analyse ${ }^{\mathbf{2 1}}$, se tournent de manière privilégiée vers cette petite enfance où se construit le futur adulte. "Géologie " est ainsi aimanté par ce séjour de l'enfance que l'âme cherche à se remémorer, non sans quelque nostalgie : "Car l'âme habite au paysage de l'enfance / et ne peut le quitter sans vieillir. Qu'ai-je fait ? / Où est ma paix ? Où sont les matins d'excellence, / l'enfant pieux portant son Dieu dans la gaieté / comme une odeur de foin, la cerise à l'oreille? » (PC, p. 18). Enfance perdue, donc, que le poète se charge de retrouver en taillant "dans l'épaisseur des mots la jeunesse du verbe " (PC, p. 18). Plus loin dans Géologie, trois " chemins " viennent ouvrir les voies de l'origine : "Chemin des sources " à travers le souvenir d'une " petite fille au goût de foin, petite fille au goût d'église "dont le poème retrouve intacts "les yeux bleus très anciens " (PC, p. 41) ; "Chemin d'Héraclite " qui désigne, à l'aurore de la pensée grecque, un " chanteur des rues, portant l'enfant soleil dans [son] oreille de sourd " (PC, p. 42) ; "Chemin d'enfance " enfin, où le sujet lyrique, sous le rythme de l'alexandrin, entend toujours ses " pas d'enfant / Les pas tremblants dans le grenier du mal de naître. " (PC, p. 42).

L'Escalier bleu, lui aussi, est traversé par toute une archéologie de l'enfance qui trouve son siège mythique dans " l'ancienne demeure / où j'ai vécu parmi les chambres familières / l'amour du monde avant sa chute dans le froid " (PC, p. 65). La parole, ici, se laisse entraîner dans la véritable spirale temporelle que constitue un escalier fait de la "pierre de l'homme originel " (PC, p. 66) et relié à " un très vieux peuple " descendant " de plus loin que l'eau du baptême " (PC, p. 67) : le motif de l'escalier conjoint ainsi la rêverie primitiviste et le souvenir de

20 Geneviève Henrot, Henry Bauchau poète, op. cit., p. 46. Sur la question du temps, voir par ailleurs le chapitre II de cet ouvrage ("Natif de mes ruines surgissantes », p. 43-77), qui apporte des analyses essentielles. 21 Sur le rapport plus général entre poésie et psychanalyse chez Bauchau, voir Marie-Claire Boons, "Henry Bauchau, le rêveur, le poète ", dans Marc Quaghebeur (dir.), Les Constellations impérieuses d'Henry Bauchau, Bruxelles, AML Éditions / Éditions Labor, 2003, p. 29-43. 
l'enfance. La deuxième section du recueil, "La maison du temps ", prolonge cet imaginaire architectural en parcourant le labyrinthe des pièces et des matériaux mis au jour dans le terreau de l'inconscient : ces " matières ingénues du cour " qui forment les murs de l'édifice de la psyché ("Les matières ", PC, p. 71) ou encore cette " matière d'enfance emmêlée de ciment " "Les chambres ", PC, p. 73), mais aussi ces "fenêtres / dans la maison du temps où mûrissaient des pommes " (" La demeure ", PC, p. 72), ces " murs des remises " sous lesquels étaient plantées des "pensées / qui regardaient le temps de la terre sans feuilles " (Les pensées ", $\mathrm{PC}$ - p. 77) ou ces " chambres d'histoires " où le père donnait voix à ses talents de conteur ("Le conteur ", PC, p. 77). Enfance originelle qui dessine la voie d'une genèse toujours recommencée, et qui éclaire à sa manière l'oracle formulé à la fin de La Sourde Oreille : "La poésie te dit qu'il ne faut pas mourir » (PC, p. 246).

C'est que, par-delà l'enfance, la poésie d'Henry Bauchau se montre attentive à une origine bien plus primitive, anténatale et pour tout dire mythique : origine même du temps qui représente peut-être le "Vrai temps ", selon le titre d'un poème de L'Escalier bleu consacré à l'évanescence de cette " plus ancienne pensée / cette idée de bien avant moi "(PC, p. 82). À la suite de ce texte, deux autres poèmes creusent la pensée de l'origine en questionnant un "Temps natal " situé dans "les nœuds de l'ancienne matière " (PC, p. 83), et un "Temps jeune " s'affirmant dans l'éveil viril du "vivant membre du plus beau mâle / d'amour prenant la belle épouse des années " (PC, p. 84). Partie en quête du temps de la naissance, cette poésie en arrive ainsi à penser la naissance du temps - peut-être, au fond, parce qu'Henry Bauchau attribue à la poésie elle-même une affinité particulière avec l'originel, l'archaïque, le pré-historique. C'est du moins ce qu'affirme "La circonstance éclatante ", justifiant ainsi le choix initial de la poésie : "parce qu'elle vient de plus loin, d'une étendue plus profonde de l'histoire et de la préhistoire humaine. Parce qu'elle me reliait à des couches plus originelles de ma géologie personnelle. » (EE, p. 20).

La suite de l'extrait montre pourtant que ce retour vers l'origine ne doit pas consister en une tentation régressive, mais au contraire en une invitation à poursuivre plus avant sur la route : "Beaucoup plus tard, je me suis aperçu que c'est en creusant dans son passé qu'on ouvre la voie de son futur. " (EE, p. 20). C'est dire, au fond, combien la poésie bauchalienne vise moins l'origine comme un refuge que comme une relance, poussant toujours plus loin ce que Le Présent d'incertitude, dans le sillage de Louis-René des Forêts ${ }^{\mathbf{2 2}}$, nomme "la douleur de naître "(PI, p. 121). Mais l'épreuve de cette naissance comporte aussi sa part de plénitude. Car il arrive que la remontée vers l'origine permette d'atteindre un horstemps à partir duquel toute une vie et toute une chronologie peuvent être considérées comme depuis un surplomb rayonnant, et rappelées dans l'instantanéité d'une présence. Cette forme d'extase temporelle, pour reprendre les termes de

22 "Dis-toi qu'aux deux extrémités du parcours / C'est la douleur de naître la plus déchirante ", écrit des Forêts dans Poèmes de Samuel Wood, Fontfroide-le-Haut, Fata Morgana, 1988, p. 19. 
Heidegger, est nettement formulée dans le Journal d'Antigone, qui décrit ainsi la marche du poème : "Il me découvre, il s'inscrit dans la force et l'illusion du temps, il ouvre le futur, le passé, il me fait saisir le présent et parfois la présence. " (JA, p. 169). "Dépendance amoureuse du poème " dit aussi la grâce particulière de ce moment où le poème naissant allie la poursuite du passé à celle du futur : "C'est un moment de bonheur où je communique avec une profondeur, avec un passé, tout en me dirigeant, de façon imprécise mais certaine, en avant. " (PC, p. 7).

C'est pourquoi, de manière récurrente, les poèmes d'Henry Bauchau usent de l'instant comme tremplin vers une synthèse où le passé, le présent et le futur s'impliquent, voire se confondent. Il se produit alors ce qu'un poème de La Chine intérieure nomme "La traversée du temps " (PC, p. 200) : traversée mémorielle qui, en l'occurrence, part des "voiles mouillées des pêcheurs de Nieuport " pour remonter jusqu'aux " chasseurs au temps de Bruegel " et se résorber dans "La fin du jour, la nuit prochaine et l'aube où des enfants vont naître ". Certains poèmes en arrivent même à neutraliser les temps en évitant l'emploi de verbes conjugués (qui impliqueraient une actualisation temporelle), et en utilisant la forme verbale impersonnelle du participe présent (comme dans la série de poèmes qui ouvrent "Liant déliant ", dernière section de L'Escalier bleu, PC, p. 91-98) ou même des énoncés nominaux (comme dans la "Demeure pour une mémoire » de Célébration, PC, p. 147). Il se pourrait, pourtant, que cette communion, ou du moins cette communication des temps ne soit accordée que comme une grâce passagère : c'est par exemple ce que suggère "Écrit pour le ciel ", qui entrevoit dans un moment ultime "L'état où nous serons, où nous sommes, où nous serons toujours " (PC, p. 179).

Car en parcourant l'éventail du temps à partir de la circonstance vécue et de l'écoute obscure du corps, le poème touche, sinon à l'éternité, du moins à une intemporalité qui dépasse le cadre humain. Associant les strates de l'inconscient à celles de l'écorce terrestre, "Géologie " guette le torrent de l'origine en se demandant : "Va-t-il plonger dans l'autre dimension du temps / où nos années seront instants de millénaires? " (PC, p. 14). De manière analogue, le locuteur du "Petit jour " (PC, p. 171-173) ne rappelle " le point du jour où j'aurai soixante ans " que pour mieux le relier à la matière de Bretagne des récits arthuriens, ainsi qu'aux figures apostoliques de Jacques le Majeur et de Jacques le Mineur, inventant même à l'occasion l'image d' "un Christ aux cheveux blancs ". La dimension religieuse de cette transmutation du temps est encore plus nette avec la suite des " Heures " dans La Pierre sans chagrin, où le rythme des heures canoniales transforme "le temps utile " des "saisons manuelles " en un " grand rituel " qui défriche et fait germer l'existence (PC, p. 134), et où la "syllabe chantée / ou l'aventure psalmodiée » parviennent à ressusciter Dieu et à rendre son brillant à "la sombre musique du temps" (PC, p. 136). Le dernier poème de la série, "Complies ", ressaisit ce mouvement du temporel vers le spirituel à travers le motif de la neige " tombée tout le jour ", et dont le " cercle de flocons " parvient à isoler un espace propice à une méditation sur "l'hiver qui plonge hors du temps " : l'heure de complies, celle du 
crépuscule et " du dernier psaume ", loin de correspondre à celle de la mort, renvoie bien plutôt à un cycle de vie perpétué par le jeûne, le silence et la prière ${ }^{\mathbf{2 3}}$ de l'abbaye du Thoronet.

Fertile alliance des contraires, la poésie bauchalienne choisit de se lier au temps vécu pour mieux se délier d'un temps mortel - d'accepter le devenir pour en faire le point de départ d'une recherche toujours recommencée de l'origine. Il y a là, au fond, un pari sur la force heuristique de cette "métamorphose " dans laquelle la fin de La Grande Troménie appelle à " entrer " (PC, p. 252), métamorphose qui, à en croire le commentaire du Journal d'Antigone sur ce passage, "suppose un changement dont nous ne sommes plus les pilotes " (JA, p. 429). La circonstance, pour reprendre ici le chemin d'Héraclite et de son panta rhei, agit dès lors comme une invitation à plonger dans le fleuve du Temps, mais un fleuve où l'amont serait paradoxalement situé en aval. Un fleuve, peut-être, analogue à cette Vienne qu'Henry Bauchau a si souvent contemplée à la lumière du soleil levant, et qui semble, à en croire le "Deuxième exercice du matin ", lui avoir appris l'" Écriture sur le sable " (PC, p. 315) : manière de suggérer combien, en se vouant à la mémoire de l'origine, la création poétique prend le risque de l'oubli et de l'effacement. La réflexion d'Henry Bauchau sur ce vers, dans "Linnocence de l'oreille ", montre du reste combien cette instabilité fragilise l'écriture : "Écrire sur le sable, c'est bien ce qu'on vit chaque jour dans le poème : les pensées, les mots, les sons, les images apparaissent, se transforment, s'inversent et s'effacent sans cesse. "(EE, p. 154). Des poèmes, pourtant, naissent et demeurent comme autant de défis à la mort - comme si Bauchau, sans le formuler mais en lui donnant corps, avait suivi le précepte donné en 1930 par Éluard et Breton dans L'Immaculée conception : "Écris l'impérissable sur le sable. ”24

Olivier Belin

Université de Cergy-Pontoise

23 Sur le rôle de la prière comme exercice de la parole et sa convergence avec l'écriture, voir Myriam Watthee-Delmotte, "Henry Bauchau : poésie et prière ", dans Pierre Halen, Raymond Michel \& Monique Michel (dir.), Henry Bauchau, une poétique de l'espérance, Berne, Peter Lang, "Recherches en littérature et spiritualité "2004, p. 219-233.

24 André Breton, Euvres complètes, tome I, Paris, Gallimard, "Bibliothèque de la Pléiade ", 1988, p. 883 . 